\title{
Preparation and Characterization of Polyhedral Octaphenylsilsesquioxane Modified Castor oil based Polyurethane
}

\author{
Yue Wen ${ }^{1}$, Pingping Jiang ${ }^{1^{*}}$, Jie Huang ${ }^{1}$, Agus Haryono ${ }^{2}$, Jianneng Deng ${ }^{3}$, Zhiliang Cao ${ }^{3}$ \\ ${ }^{1}$ The Key Laboratory of Food Colloids and Biotechnology, Ministry of Education, School of Chemical and Material \\ Engineering, Jiangnan University, Wuxi 214122, China. \\ ${ }^{2}$ Polymer Chemistry Group, Research Center for Chemistry, Indonesian Institute of Sciences (LIPI), Kawasan \\ Puspiptek, Serpong, 15314, Indonesia \\ ${ }^{3}$ Nantong Hairma Technology Co., LTD, Nantong 226000 \\ *Corresponding author : ppjiang@jiangnan.edu.cn
}

A R T I C L E I N F O
Article history:
Received date : 21 May 2018
Revised date : 25 July 2018
Accepted date: 8 August 2018
$\begin{aligned} & \text { Available online at : } \\ & \text { http://inajac.lipi.go.id }\end{aligned}$
Keywords:
$\begin{aligned} & \text { Castor oil, hybrid } \\ & \text { polyurethane, Polyhedral } \\ & \text { Octaphenylsilsesquioxane. }\end{aligned}$

\begin{abstract}
Vegetable oil-based polyurethanes were synthesized using castor oil (CO) as feedstock, which were environment friendly and renewable. To compensate for shortcomings of these materials, a series of polyurethane/polyhedral octaphenylsilsesquioxane (OPS) hybrids with different OPS contents were prepared by physical mixing in the solutions. Chemical structure, morphology and thermal properties of these hybrids were characterized through Fourier transform infrared spectrometer (FTIR), scanning electron microscope (SEM), differential scanning calorimetry (DSC), thermal gravimetric analyzer (TGA), tensile test techniques and static contact angle. The results show that the hybrid polyurethanes display both enhanced glass transition temperatures $(\mathrm{Tg})$, initial decomposition temperature $\left(\mathrm{T}_{\mathrm{d} 5}\right)$ and tensile strength with low OPS contents. While with high contents, these values decline with the severe aggregation of nano-particles as shown in the SEM images. Meanwhile, the hybrid polyurethanes displayed enhanced surface hydrophobicity as the contact angle with water revealed.

(C) 2018 Indonesian Journal of Applied Chemistry. This is an open access article under the CC BY-NC-SA license (https://creativecommons.org/licenses/by-nc-sa/4.0/).
\end{abstract}

\section{INTRODUCTION}

Combining inorganic or organometallic segments into organic polymers is an effective approach to obtain organic-inorganic hybrid materials with excellent and comprehensive properties, which represents new chemical technology for the development of new materials [1-5]. Polyhedral oligomeric silsesquioxanes (POSS) is composed of two cages, one cage inorganic - like $\mathrm{Si}-\mathrm{O}$ framework, and the other organic - like groups covalently bonded to each $\mathrm{Si}$ atom, one or more of which is reactive. They have drawn widening interest due to the well-defined nano-sized cage-like structure and possible use as precursors features [6]. There have been a great number of studies focused on the POSScontaining organic-inorganic hybrids in the past years [7-10]. The functionality of POSS macromers possess a variety of approaches to be designed and utilized. Specifically, the functionality of POSS is roughly divided into three categories, monofunctional POSS macromers, difunctional POSS macromers and multifunctional POSS macromers. Copolymerization or reactive grafting techniques are utilized to introduce single reactive groups to the organic polymers to obtain the monofunctional POSS macromers. Alternatively, single polymerizable groups were capped to the ends of polymer chains to get the so-called POSS - capped monofunctional POSS macromers [11-15]. POSS cages behave as either end groups and pendent side groups in these organic-inorganic hybrids, and keep the main chains of organic polymers remain unchanged. Besides, multifunctional POSS macromers have also been incorporated into polymers to form the POSS-containing nanocomposites such as poly (ethylene imine) [16], polyimide [17], 
polymethyl methacrylate [18], polyurethane [19-21], polybenzoxazines [22,23] and epoxy [24-30]. With the multiple chemical bonds between polymers and the POSS macromers, POSS cages act as bulky nano-crosslinkers to participate in the formation of polymer networks so these organic-inorganic systems possess three dimensional cross-linked structures.

Polyurethanes (PUs) are a significant class of materials, and their various applications force them achieve higher requirements. In addition, the increasing price of crude oil and environmental concerns have triggered great attention on the renewable resources, which are emerging as interesting sustainable platform to design and develop eco-friendly functional polymers. Vegetable oils (CO?) such as cotton seed, soybean, castor, linseed, etc. are important sources for the development of polymers, which also offer excellent properties. Among them, castor oil (CO) equipped the unique backbone chemistry makes it the material of choice for the development of polyurethanes. Besides, due to its inherent hydroxyl functionality, $\mathrm{CO}$ serves as a natural polyol for the preparation of polyurethane. Furthermore, the castor oilbased polyurethanes provided flexibility and water resistant properties from the long fatty acid chain in $\mathrm{CO}$ backbone [37-39]. However, vegetable oil-based polyurethanes possess relatively low rigidity, thermal stability, and strength required for structural applications by themselves. Therefore, large amount of investigations were performed on the modification of the vegetable oil-based polyurethane by the use of polysiloxanes and
POSS. For instance, Matisons [40] prepared octa(isocyanate) - functionalized POSS macromere, and then utilized to prepare organic-inorganic hybrid polyurethane. Choudhury and Oaten [41] and Turri and Levi $[42,43]$ investigated the surface properties of linear polyurethanes modified with monofunctional POSS macromers; and found that with the presence of POSS, the surface free energy of the materials significantly reduced and the surface hydrophobicity of the materials improved. Mather and co-workers [44] synthesized poly( $\varepsilon$-caprolactone)-POSS with the different molar ratio of PCL:POSS. In addition, other POSS equipped with active groups such as amino-group and hydroxylgroup were also successfully grafted to polyurethane chains to pursuit higher quality of material $[45,46]$.

In As described above, most of the approaches developed hybrid materials by adding external organosilane coupling groups. However, physical mixing is also a way to incorporate POSS molecules into polymers except chemical introduction like grafting, cross-linking, and copolymerization [47]. Compatibility and thermostability are important parameters of physical mixing. Polyhedral octaphenylsilsesquioxane have high thermal stabilities and can be used in the field of flame retardants and high-temperature polymers [48]. In our attempt to find new hybrid materials polyhedral octaphenylsilsesquioxane and use of $\mathrm{CO}$ as renewable resources to synthesize sustainable polyurethane. The route not only solve the global emergency environmental problems but
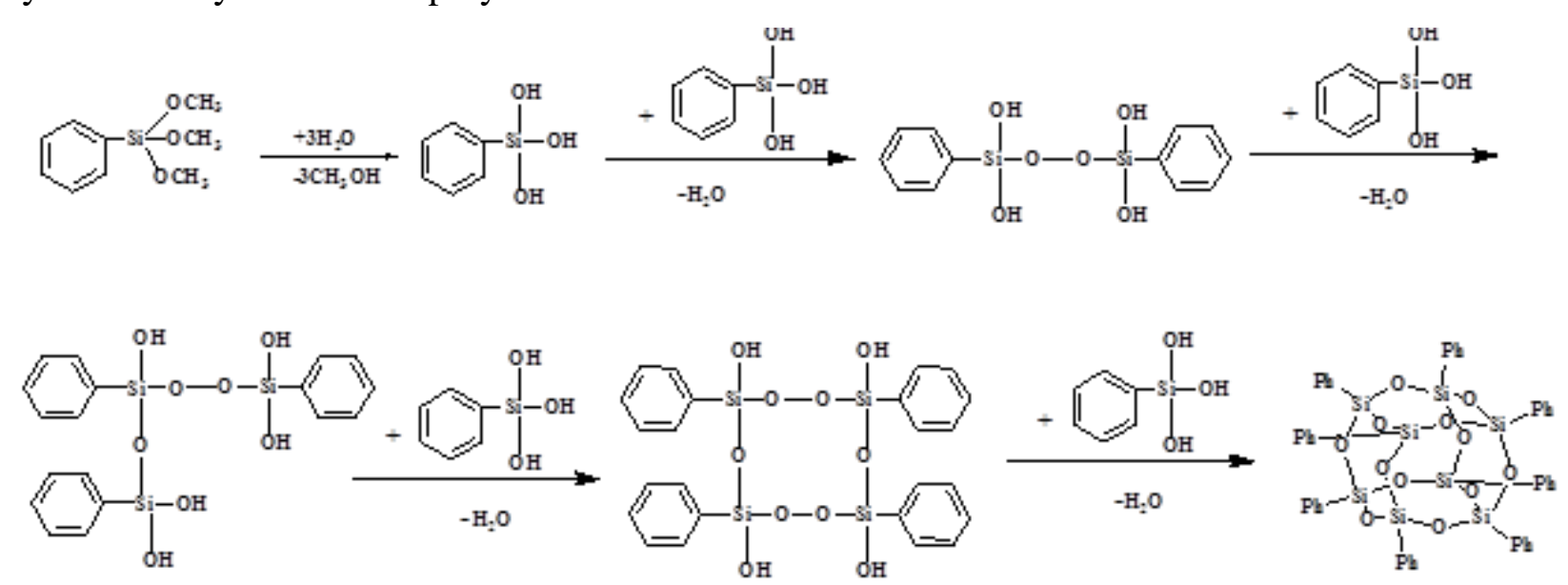
Table 1. Chemical composition of pure polyurethane and hybrid polyurethanes.

\begin{tabular}{cccccc}
\hline Samples & $\begin{array}{c}\text { CO mass } \\
(\mathbf{g})\end{array}$ & $\begin{array}{c}\text { IPDI vol. } \\
(\mathbf{m l})\end{array}$ & $\begin{array}{c}\text { Stannous octoat vol. } \\
(\boldsymbol{\mu l})\end{array}$ & $\begin{array}{c}\text { OPS mass } \\
(\mathbf{g})\end{array}$ & $\begin{array}{c}\text { OPS } \\
(\mathbf{w t .} \%)\end{array}$ \\
\hline Pure PU & 4.00 & 3.05 & 100 & 0 & 0 \\
Hybrid-1 & 4.00 & 3.05 & 100 & 0.01 & 0.13 \\
Hybrid-2 & 4.00 & 3.05 & 100 & 0.03 & 0.38 \\
Hybrid-3 & 4.00 & 3.05 & 100 & 0.05 & 0.62 \\
Hybrid-4 & 4.00 & 3.05 & 100 & 0.07 & 0.87 \\
Hybrid-5 & 4.00 & 3.05 & 100 & 0.09 & 1.14 \\
\hline
\end{tabular}

also overcome the shortcoming of pure vegetable oil-based polyurethanes. Toward this end, we reported the synthesis of polyhedral octaphenylsilsesquioxane (OPS) firstly. Thereafter, different content of OPS was mixed in the polyurethane through ultrasound shaking. Meanwhile, we also discussed the thermal properties, mechanical properties, the surface properties and morphology of these hybrid polyurethanes. The results of this paper can provide some insight and scientific data for vegetable oilbased hybrid polyurethanes.

\section{EXPERIMENTAL SECTION}

\subsection{Materials}

Castor oil (CO) was purchased from Shanghai Reagent Co. and stored with $4{ }^{\circ} \mathrm{A}$ molecular sieves. Phenyltrimethoxysilane (98\%) was purchased from Chengdu Xiya Reagent Co. and used without further purification. Isophorone diisocyanate (IPDI) was obtained from Wuxi East Grace Electronic Material Technology Co., Ltd.. Other reagents such as tetrahydrofuran (THF) was refluxed above metal sodium and then distilled and stored in the presence of a $4{ }^{\circ} \mathrm{A}$ molecular sieve of molecular sieves. Ethyl acetate (EtAc) was dehydrated then stored in the presence of $4{ }^{\circ} \mathrm{A}$ molecular sieves. Other reagents in this article were also purchased from Shanghai Reagent Co., China and used as received.

\subsection{Synthesis}

\subsubsection{Synthesis of polyhedral octaphenylsilsesquioxane (OPS)}

The structure and synthetic route to polyhedral octaphenylsilsesquioxane was shown in Scheme 1. The detailed synthetic methods were as follows: phenyltrimethoxysilane (12 g, $0.06 \mathrm{~mol})$ and anhydrous ethanol $(150 \mathrm{~mL})$ were added to the three-neck flask. Next, distilled water $(1 \mathrm{~mL})$ was added dropwise to the solution at the temperature of $30^{\circ} \mathrm{C} \sim 40^{\circ} \mathrm{C}$, and the system was stirred for about $2 \mathrm{~h}$. Then the quantitative tetramethyl ammonium hydroxide in ethanol solution was slowly dropped to the reaction system for several times, and reacted for about another $24 \mathrm{~h}$ under nitrogen atmosphere. After reaction, filtered, washed and dried, tetrahydrofuran and ethanol were used as a solvent for recrystallization purification of mixed solution. White powder was obtained as product.

Table 2. TGA results of pure polyurethane and hybrid polyurethanes.

\begin{tabular}{lcccc}
\hline Samples & $\begin{array}{c}\text { OPS } \\
(\mathbf{w t \%})\end{array}$ & $\begin{array}{c}\boldsymbol{T}_{\mathbf{d} 5} \\
\left({ }^{\circ} \mathbf{C}\right)\end{array}$ & $\begin{array}{c}\text { Char } \\
\text { Residue } \\
(\mathbf{w t} \%)\end{array}$ & $\boldsymbol{T}_{\mathbf{g}}\left({ }^{\circ} \mathbf{C}\right)$ \\
\hline Pure PU & 0 & 212 & 0 & 25.08 \\
Hybrid-1 & 0.13 & 223 & 1.03 & 27.64 \\
Hybrid-2 & 0.38 & 239 & 1.58 & 30.57 \\
Hybrid-3 & 0.62 & 256 & 2.05 & 35.42 \\
Hybrid-4 & 0.87 & 234 & 2.34 & 31.10 \\
Hybrid-5 & 1.14 & 221 & 2.68 & 32.64 \\
\hline
\end{tabular}

\subsubsection{Preparation of castor oil based hybrid polyurethanes modified with OPS}

The hybrid polyurethanes were prepared using the formulation as summarized in Table 1. Castor oil was further reacted with IPDI in presence of $0.01 \mathrm{wt} \%$ of stannous octoate as catalyst at around $80{ }^{\circ} \mathrm{C}$ to get $-\mathrm{NCO}$ terminated pre-polymers. The prepared -NCO terminated hybrid pre-polymers were further reacted with chain extender for the preparation of final hybrid polyurethanes. Typically, synthetic procedure was as follows: Castor oil (4 g, $11.64 \mathrm{mmol} \mathrm{OH})$, and IPDI 
(3.05ml) were charged into a flask equipped with a magnetic stirrer. Ethyl acetate (EtAc) $15 \mathrm{~mL}$ and stannous octoate $(100 \mathrm{uL})$ were added to the mixture, then the reactant mixture was slowly heated up to $80^{\circ} \mathrm{C}$ and maintained at $80^{\circ} \mathrm{C}$ under nitrogen atmosphere for $2 \mathrm{~h}$ with vigorous stirring. After the reaction, -NCO terminated pre-polymer was obtained, and the content of isocyanate (NCO) was determined followed ASTM D5155-96. Different contents of OPS (See Table.1) and stoichiometric amount of chain extender 1, 4butanediol (BDO) were then added to the reaction mixture, and then the mixture was mixed under supersonic vibration for $1 \mathrm{~h}$. Finally, the resultant mixture was poured into a Teflon plate, and kept at atmospheric moisture and laboratory humidity condition to evaporate the solvent and then cured at $80{ }^{\circ} \mathrm{C}$ for $48 \mathrm{~h}$.

Table 3. Static contact angles of pure polyurethane and hybrid polyurethanes.

\begin{tabular}{|c|c|c|c|}
\hline \multirow{2}{*}{ Samples } & \multirow{2}{*}{$\begin{array}{c}\text { OPS } \\
\text { (wt.\%) }\end{array}$} & \multicolumn{2}{|c|}{ Static contact angle } \\
\hline & & $\theta_{\mathrm{H} 2 \mathrm{O}}(\mathrm{deg})$ & $\begin{array}{c}\theta_{\text {ethylene glycol }} \\
\text { (deg) }\end{array}$ \\
\hline Pure PU & 0 & $80.0 \pm 1.23$ & $48.1 \pm 0.60$ \\
\hline Hybrid-1 & 0.13 & $83.6 \pm 1.04$ & $52.2 \pm 1.08$ \\
\hline Hybrid-2 & 0.38 & $88.6 \pm 1.02$ & $61.3 \pm 1.09$ \\
\hline Hybrid-3 & 0.62 & $91.7 \pm 1.35$ & $68.4 \pm 1.24$ \\
\hline Hybrid-4 & 0.87 & $94.3 \pm 1.26$ & $72.3 \pm 1.20$ \\
\hline Hybrid-5 & 1.14 & $101.0 \pm 1.14$ & $75.2 \pm 1.34$ \\
\hline
\end{tabular}

\subsection{Measurements}

${ }^{1} \mathrm{H}(400 \mathrm{MHz}) \mathrm{NMR}$ spectra were obtained on A Bruker Instruments (model Avance 400, Germany), and $\mathrm{CDCl}_{3}$ was used as solvent. For MALDI-TOF MS analysis, The matrix 2,5dihydroxybenzoic acid, dissolved in THF (50 $\mathrm{mg} / \mathrm{mL}$ ) were mixed with polyhedral octaphenylsilsesquioxane solution $(0.1 \mathrm{mg} / \mathrm{mL}$ in $1: 1 \mathrm{v} / \mathrm{v}$ ratio). The resultant solution was deposited on a stainless steel sample plate and dried. The ATR-FTIR spectra were obtained from Nicolet 6700 infrared spectrometer. All spectra were measured between $4000 \mathrm{~cm}^{-1}$ to $500 \mathrm{~cm}^{-1}$ with averaging 32 scans at a resolution of $4 \mathrm{~cm}^{-1}$. The morphology of all the hybrid polyurethanes were observed by scanning electron microscopy (S-4800, Hitachi), before test, all the samples were fractured with liquid nitrogen and coated with gold. The thermodynamic properties of the hybrid polyurethanes were measured by differential scanning calorimetry (DSC-8000) and thermogravimetric analysis (TGA/1100SF), respectively. The thermogravimetric analysis (TGA) was from $35^{\circ} \mathrm{C}$ to $600{ }^{\circ} \mathrm{C}$ ramped at $20^{\circ} \mathrm{C}$

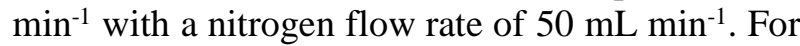
DSC analysis, samples (5-10 $\mathrm{mg}$ ) underwent a temperature range from $-80^{\circ} \mathrm{C}$ to $150^{\circ} \mathrm{C}$ at a

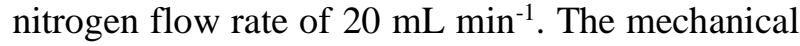
properties were measured according to ASTM D $882-97$ on a tensile tester model with $10 \mathrm{~mm} / \mathrm{min}$ extension rate and the gauge length was $4 \mathrm{~mm}$, and five specimens were used for each sample. The surface hydrophobicity of the hybrid polyurethanes were analyzed on a DCA-315 static contact angles. Ultrapure water and glycol were chosen as probe liquids at room temperature. All samples were tested at three different positions and the results were expressed as mean value.

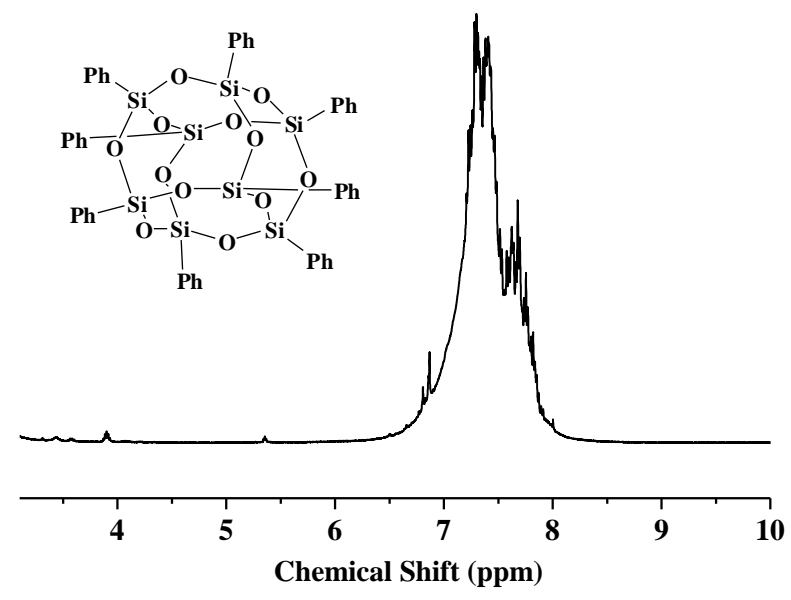

Fig.1. ${ }^{1} \mathrm{H}$ NMR of OPS

\section{RESULTS AND DISCUSSION}

\subsection{Synthesis}

\subsubsection{Synthesis of polyhedral octaphenylsilsesquioxane (OPS)}

To further confirm the successful synthesis of polyhedral octaphenylsilsesquioxane (OPS), ${ }^{1} \mathrm{H}$ NMR and MALDI-TOF-MS were utilized to characterize the formation. ${ }^{1} \mathrm{H}$ NMR were carried out and shown in Fig.1. The resonance assignable to the protons of phenyl groups were detected at 7-8 ppm. Concurrently, polyhedral octaphenylsilsesquioxane (OPS) was subjected to MALDI-TOF mass spectroscopy to measure its molecular weight and the mass spectrum was presented in Fig. 2. It was seen that the OPS possessed a molecular weight of $\mathrm{M}=1033.51$ (viz. 1056.51-23), 
which was assignable to the value calculated according to the structural formula (see Scheme 1). The results of ${ }^{1} \mathrm{H}$ NMR and MALDI-TOF-MS indicated that polyhedral octaphenylsilsesquioxane (OPS) was successfully obtained.

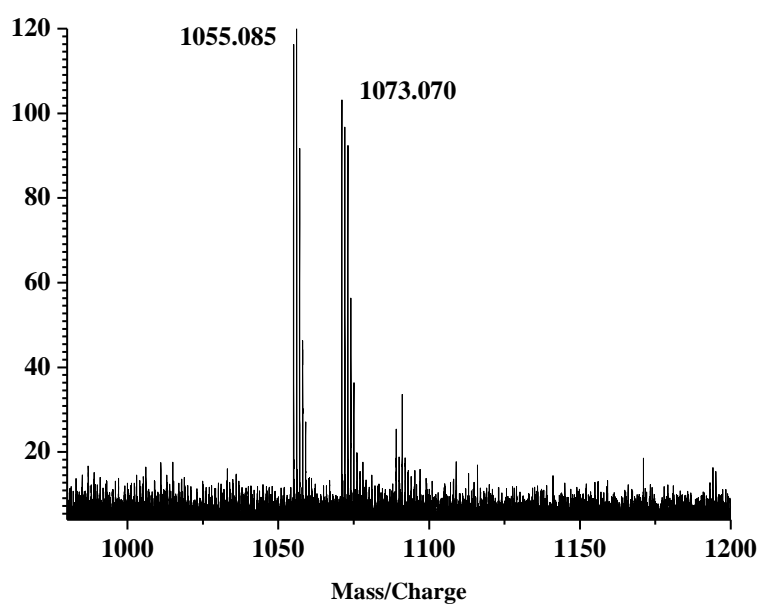

Fig.2. MALDI-TOF-MS of OPS

\subsubsection{Synthesis of hybrid polyurethanes}

The route of synthesis for the hybrid polyurethanes modified with OPS was as follows: In the first step, the pre-polymer of hybrid polyurethane was synthesized via the reaction of IPDI and CO. Thereafter, the chain extenders were added to achieve the chain extension reaction, and at the same time, polyhedral octaphenylsilsesquioxane (OPS) was added through supersonic vibration to the solution with different content. By controlling the addition of OPS, the hybrid polyurethanes were obtained with a content of OPS up to $1.14 \mathrm{wt} \%$. Representatively shown in Fig. 3 were the IR spectra of pure polyurethane and hybrid polyurethanes. Peaks around $3340 \mathrm{~cm}^{-1}$ and $1560 \mathrm{~cm}^{-1} \sim 1520 \mathrm{~cm}^{-1}$ belonged to the characteristic absorption peaks of - $\mathrm{NH}-$, which all the polyurethanes exhibited. The stretching vibration peaks of $-\mathrm{CH}_{3}$ - and $-\mathrm{CH}_{2}$ - in the castor oil chain appeared at $2930 \mathrm{~cm}^{-1}$ and $2850 \mathrm{~cm}^{-1}$. Besides, two peaks around 1130 and $1030 \mathrm{~cm}^{-1}$ were derived from the antisymmetric stretching vibrations and asymmetric stretching vibrations of $\mathrm{Si}-\mathrm{O}-\mathrm{Si}$ bonds. Unfortunately, for the hybrid polyurethanes, the overlap between the aliphatic ether and Si-O-Si groups led to small difference in the IR spectra [51]

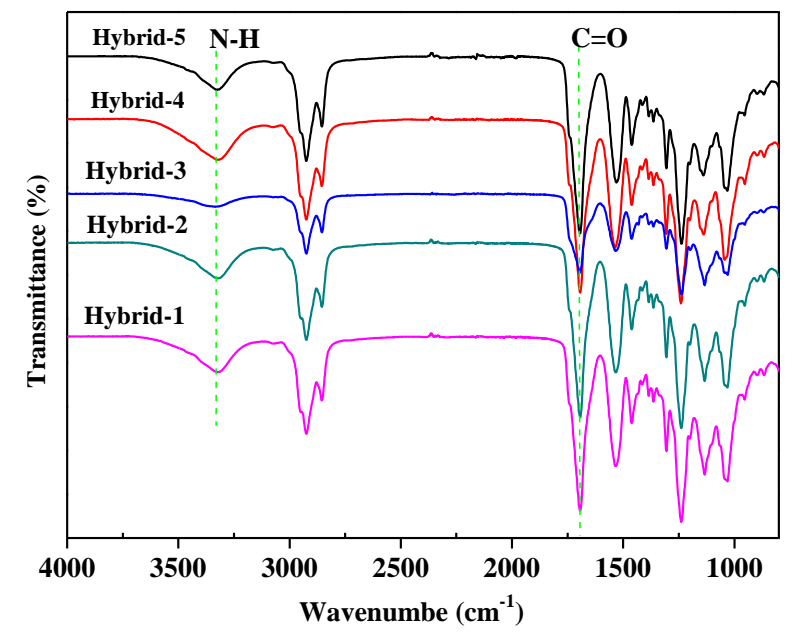

Fig.3. IR spectra of hybrid polyurethanes.

\subsection{Thermal decomposition behaviour}

Thermogravimetric analysis (TGA) was utilized to evaluate the thermal stability of the pure and hybrid polyurethanes and the TGA curves were shown in Fig.4. It was indicated that all hybrid polyurethanes displayed similar degradation profiles with the pure polyurethane, suggesting that the incorporation of OPS did not significantly alter the degradation mechanism. It was also seen that hybrid polyurethanes displayed a higher thermal stability than the pure PU in terms of the initial degradation temperatures and the yields of decomposition residues. To be convenient for comparison, $\mathrm{T}_{\mathrm{d} 5}$ was defined as the onset of decomposition temperature, where the samples attained 5\% weight loss. The $\mathrm{T}_{\mathrm{d} 5}$ of the hybrid polyurethanes were significantly higher than the pure polyurethane, suggesting that the presence of OPS retarded the randomchain scission because of the rigid structure of the OPS. Furthermore, the hybrid polyurethanes displayed higher residuals of degradation than pure polyurethane, and the yields of the degradation residues increased with increasing the content of OPS (see Table 2 ). The increased yields of degradation residues were attributed to the formation of the ceramics from OPS moiety during the thermal decomposition. To be specific, the decomposition of urethane bonds took place at 
above $200^{\circ} \mathrm{C}$. Afterwards, the second decomposition process occurred at about $340^{\circ} \mathrm{C}$ belonged to the chain scission of castor oil [52-54]. In addition, the decomposition rates of the hybrid polyurethanes during the second decomposition process were slower, due to the curves became flatter above $350^{\circ} \mathrm{C}$ with high OPS content. At high temperature, the silicon dioxide from the oxidation of OPS would be wrapped in the surface so that the release of gaseous products from segmental decomposition was suppressed, which significantly improved the thermal insulation and the flame resistance of the material $[55,56]$.

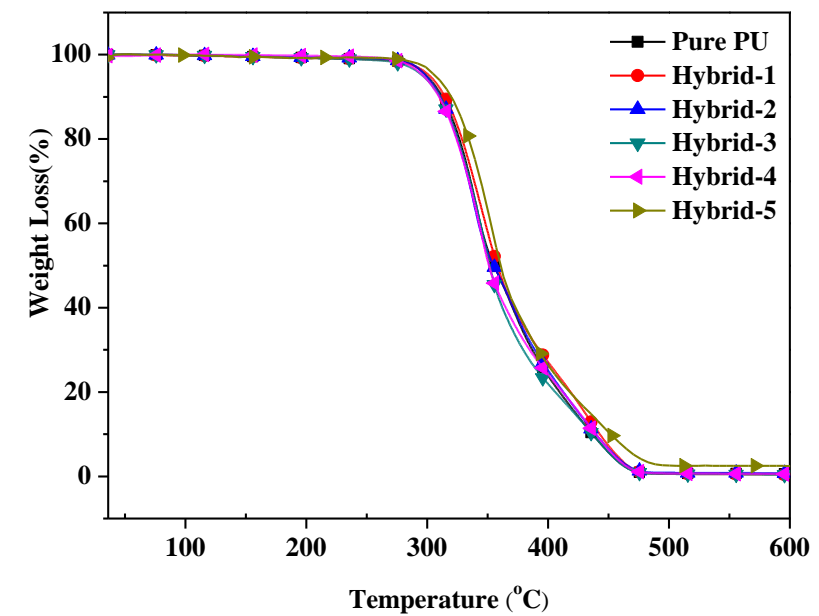

Fig.4. TGA curves of pure and hybrid polyurethanes.

\subsection{DSC Analysis}

Differential scanning calorimetry (DSC) was carried out to measure the glass transition temperature $(\mathrm{Tg})$ of all the hybrid polyurethanes, the DSC curves in the range of $-80{ }^{\circ} \mathrm{C}$ to $120{ }^{\circ} \mathrm{C}$ were shown in Fig.5., and all $\mathrm{Tg}$ (the midpoint of the stepwise decrease of the heat flow trace observed during heating) values were listed in Table 2 . It was seen that all the hybrid polyurethanes displayed single glass transitions, and the glass transition temperatures (Tg's) were varied with the content of OPS. The single Tg's indicated that all the hybrid polyurethanes were homogeneous. Notably, the $\mathrm{Tg}$ of the hybrid polyurethanes increased with the inclusion of OPS, which from $\mathrm{Tg}=25.08^{\circ} \mathrm{C}$ (pure PU) to $\mathrm{Tg}=35.42^{\circ} \mathrm{C}$ (PU/OPS with 0.62 wt.\% OPS).
But with the OPS content above 0.62 wt \%, the Tg value was decreased with the increased content of OPS. It was supposed that two opposite factors led to the result. Firstly, the inclusion of OPS cage increased the chain rigidity, which restrict the chain motion and decreased the free volume, so it needed higher temperature to reach a glass transition in the hybrid polyurethane. Secondly, serious aggregation appeared in the high OPS content, and the aggregation particles acted as solid lubricant which increased the free volume, resulted in the decrease of the $\mathrm{Tg}$.

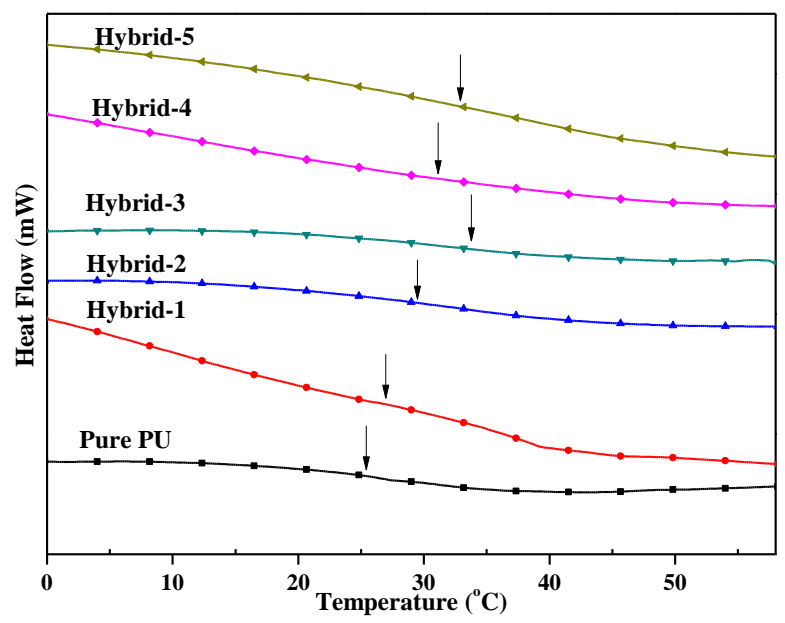

Fig.5. DSC curves of pure and hybrid polyurethanes.

\subsection{Morphology}

The hybrid polyurethanes were subjected to the scanning electron microscopy (SEM) to investigate the distribution of OPS in surface morphology. The images of the hybrid polyurethanes were shown in Fig.6. It was proposed that with the low content of OPS, the morphology of the hybrid polyurethanes displayed relatively smooth structure and the particle size distribution was uniform. However, as OPS loading increased due to the hydrophobicity of benzene ring, the dispersed particles gradually seeped into the organic matrix and small particles gradually aggregated into larger ones. Fig.6d. showed that with the $1 \mathrm{k}$ magnification times, the OPS particles was already formed an aggregates, which significantly influenced the properties of the hybrid polyurethanes. 

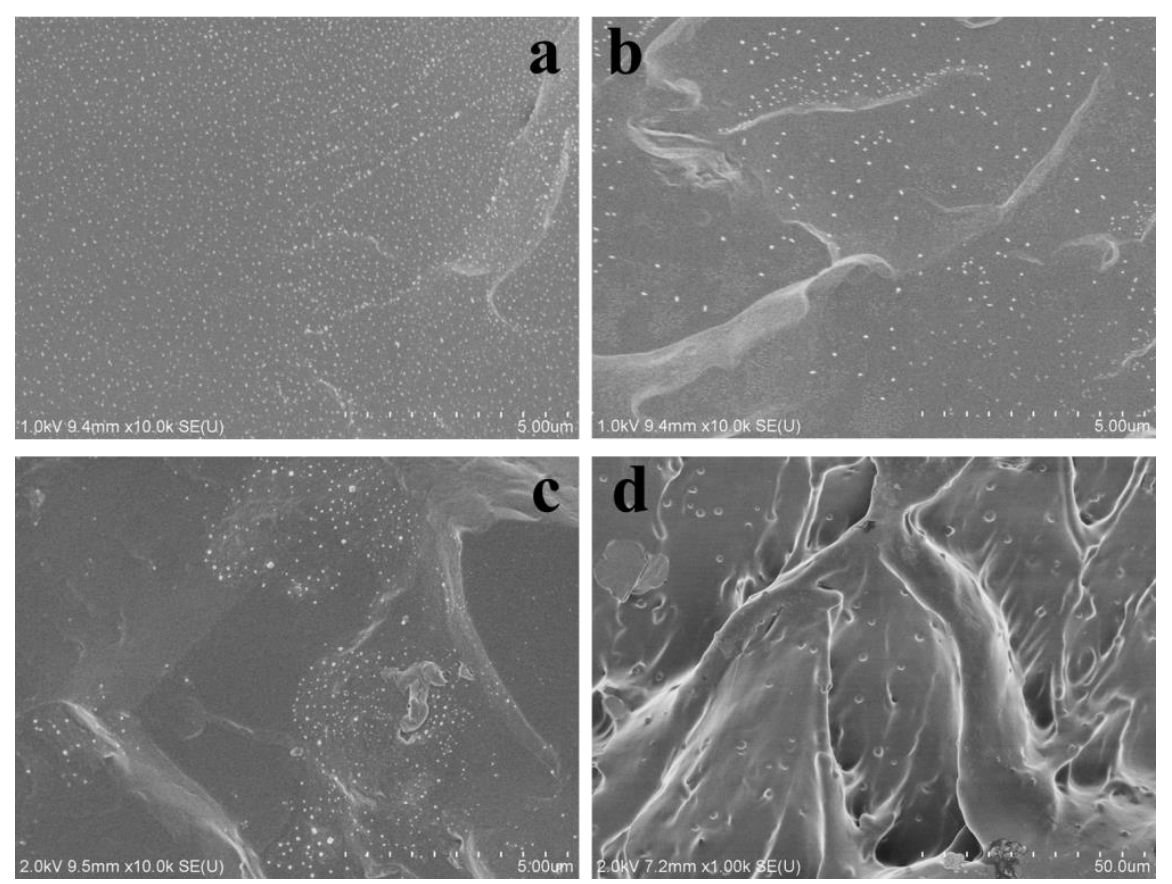

Fig.6. SEM images of hybrid polyurethanes (a) hybrid-2 (0.38 wt.\% poss). (b) hybrid-3 (0.62 wt.\% poss). (c) hybrid-4 (0.87wt.\% poss). (d) hybrid-5 (1.14 wt.\% poss ).

\subsection{Mechanical Properties of Hybrid polyurethanes}

The mechanical properties of hybrid polyurethanes were further investigated . Fig.7. showed the curves of elongation at break and tensile strength. It can be easily observed that the tensile strength was increased with increased OPS content, from $11.58 \mathrm{MPa}(0$ wt\% OPS) to $48.69 \mathrm{MPa}(0.62 \mathrm{wt} \%$ OPS $)$, it was speculated that the OPS cages on the polymeric matrices existed the force of nanoreinforcement, the interaction of the factor led to the increase of tensile strength. However, when the OPS content above $0.62 \mathrm{wt} \%$, the tensile strength was decreased with the increased OPS content. On the other hand, the elongation at break also kept decreased as the increase of the OPS content. It was proposed that when the OPS content exceeded a certain limit due to the serious aggregation, the mechanical impairment occurred at higher OPS content.

\subsection{Surface hydrophobicity}

The contact angle (CA) measurement was carried out on the pure polyurethane and the hybrid polyurethanes using water and ethylene

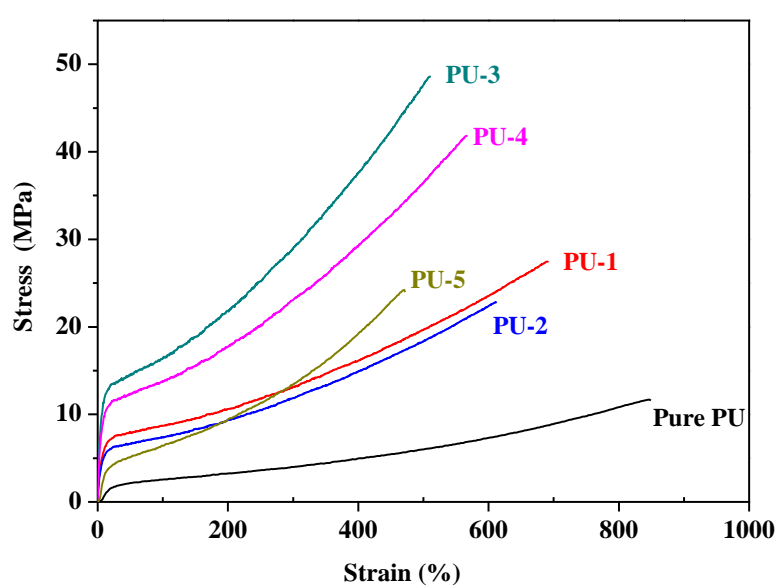

Fig.7. Tensile strength and elongation at break curves of all Pus.

glycol. Their corresponding contact angles summarized in Table 3 and Fig.8.The pure polyurethane showed the wettability properties with water contact angle of about $80.0^{\circ}$. However, with the introduction of OPS, the surface hydrophobicity of hybrid polyurethanes were increased significantly, The improvement of the hydrophobic capacity of hybrid polyurethanes could be confirmed by increasing OPS weight ratio due to the formation of Si-O-Si linkage by moisture curing process while oleophobic properties were achieved in a similar manner with ethylene glycol. Therefore, it can be concluded that the hydrophobicity and oleophobicity of 
the hybrid polyurethanes could be effectively turned by varying the feed ratio of OPS.

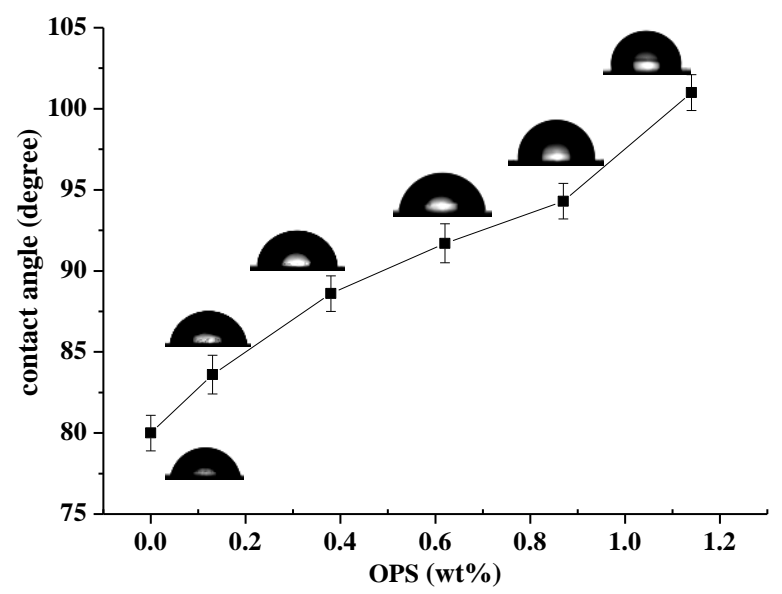

Fig.8. Plot of surface water contact angles as a function of the content of all Pus.

\section{CONCLUSION}

In this work, we successfully synthesized polyhedral octaphenylsilsesquioxane, and a series of renewable resource-based polyurethanes and their PU-OPS hybrid polyurethanes using CO, IPDI and different mole ratios of OPS. Castor oil was reacted with IPDI to get isocyanate terminated prepolymer. The excess isocyanate of the prepolymers was further reacted with BDO chain extender. Different content of OPS was added at the same time by the means of ultrasonic shaking. Hybrid polyurethanes were studied for its thermodynamic performance, mechanical properties, morphology and surface hydrophobicity using different techniques. Thermogravimetric analysis (TGA) revealed that the stability of the hybrid polyurethanes were improved in terms of the high temperatures and the yields of degradation residues. The DSC results indicated that the hybrid polyurethanes exhibited enhanced glass transition temperatures $(\mathrm{Tg})$ compared to the pure polyurethanes. The tensile strength of composites was enhanced because of OPS addition into polyurethane. The hydrophobic and oleophobic character of the hybrid polyurethanes was found to increase with an increasing OPS content. Therefore, the judicious modification of polyurethane with
OPS are critical criterion for the design of high performance polyurethane applications. Overall, we have been able to show a new strategy to design castor oil-based hybrid polyurethanes with improved thermal, mechanical and surface hydrophobicity which is expected to reduce the use of petroleum based raw materials for the development of polyurethanes.

\section{ACKNOWLEDGMENT}

This work was supported by research grants from National Key Technology Research and Development Program (2012BAD32B03-4), the Fundamental Research Funds for the Central Universities (JUSRP51623A) and the Cooperative Innovation Foundation of Industry, Academy and Research Institutes (BY2013015-10) in Jiangsu Province of China.

\section{REFERENCES}

[1]. G. M. Whitesides, J. P. Mathias and C. T. Seto, Science, 254, 1312. (1991).

[2]. T. Lan, P. D. Kaviratan and T. J. Pinnavaia, Chem. Mater., 7, 2144. (1995).

[3]. E. P. Giannelis, R. Krishnamoorti and E. Manias, Adv. Polym. Sci., 138, 107.(1999).

[4]. K. J. Shea and D. A. Loy, Acc. Chem. Res., 34, 707. (2001).

[5]. C. Sanchez, B. Julian, P. Belleville and M. Popall, J. Mater. Chem., 2005, 15, 3559. (2005).

[6]. S. Wu, M. Kakimoto and H. Oikawa. Macromolecules;41:3481-3487. (2008).

[7]. P. D. Lickiss, F. Rataboul, Adv. Organomet. Chem., 57, 1-116. (2008).

[8]. S.-W. Kuo, F.-C. Chang, Prog. Polym. Sci., 36, 1649-1696. (2011).

[9]. S. H. Phillips, d, S. J. Tomczak, Curr. Opin. Solid State Mater. Sci., 8, 2129.(2004). 
[10]. Y. Abe, T. Gunji, Prog. Polym. Sci., 29, 149-182.(2004).

[11]. K. Zeng, L. Wang, S. Zheng, J. Colloid Interface Sci., 363, 250-260. (2011).

[12]. B. S. Kim, P. T. Mather, Macromolecules, 35, 8378-8384. (2002).

[13]. B. S. Kim, P. T. Mather, Macromolecules, 39, 9253-9260. (2006).

[14]. B. S. Kim, P. T. Mather, Polymer, 47, 6202-6207. (2006).

[15]. W. Zhang, A. H. E. Mueller, Macromolecules, 43, 3148-3152. (2010).

[16]. K. Zeng, Y. Liu and S. Zheng, Eur. Polym. J., 44, 3946-3956. (2008).

[17]. J.-C. Huang, Y. Xiao, K. Y. Mya, J. Dai and Y. P. Siow, Polymer, 44, 4491-4499. (2003).

[18]. R. O. R. Costa, R. Tamaki and R. M. Laine, Macromolecules, 34, 5398-5407. (2001).

[19]. H. Liu and S. Zheng, Macromol. Rapid Commun., 26, 196-200. (2005).

[20]. D. Neumann, L. Tran and J. G. Matisons, J. Am. Chem. Soc., 124, 13998-13999. (2002).

[21]. Y. Liu, Y. Ni and S. Zheng, Macromol. Chem. Phys., 207, 1842-1851. (2006).

[22]. Y. Liu and S. Zheng, J. Polym. Sci., Part A: Polym. Chem., 44, 1168-1181. (2006).

[23]. K.-W. Huang and S.-W. Kuo, Macromol. Chem. Phys., 211, 23012311. ( 2010).

[24]. Y. Ni, S. Zheng and K. Nie, Polymer, , 45, 5557-5568. (2004).

[25]. R. M. Laine, J. Choi and I. Lee, Adv. Mater., 13, 800-803. (2001).

[26]. J. Choi, A. F. Yee and R. M. Laine, Macromolecules, 36, 5666-5682. (2003).

[27]. J. Choi, R. Tamaki, S. G. Kim and R. M. Laine, Chem. Mater., 15, 3365-3375. (2003).
[28]. J. Choi, J. Harcup, Q. Zhu and R. M. Laine, J. Am. Chem. Soc., 123, 1142011430. (2001).

[29]. J. Choi, S. G. Kim and R. M. Laine, Macromolecules, 37, 99-109. (2004).

[30]. J. Choi, A. F. Yee and R. M. Laine, Macromolecules, 37, 3267-3276. (2004).

[31]. S. Wu, M. A. Kakimoto and H. Oikawa. Macromolecules;40:5698-5705. (2007).

[32]. K. Yoshida, K. Watanabe and N. Ootake. US Pat Application 20040249103A1, 2004.

[33]. T. Kato, K. Yoshida and Y. Yamamoto. Jpn Kokai Tokkyo Koho 2006265243A, 2006.

[34]. S. Wu, M. A. Kakimoto and H. Oikawa. Macromolecules;41:3481-3487. (2008).

[35]. M. A. Hoque, S. Shinke and Y. Kawakami. Macromolecules;42:33093315. (2008).

[36]. J. Huang and P. Jiang. J Mater Sci;51:2443-2452. (2016).

[37]. D. S. Ogunniyi, Bioresour. Technol., 97, 1086-1091. (2006).

[38]. Z. S. Petrovic, Polym. Rev., 2008, 48, 109-155. (2008).

[39]. K. T. Achaya, J. Am. Oil Chem. Soc., 48, 758-763. (1971).

[40]. D. Neumann, L. Tran and J. G. Matisons, J. Am. Chem. Soc., 124, 13998. (2002).

[41]. M. Oaten and N. R. Choudhury, Macromolecules, 38, 6392. (2005).

[42]. S. Turri and M. Levi, Macromolecules, 38, 5569.(2005).

[43]. S. Turri and M. Levi, Macromol. Rapid Commun., 26, 1233. (2005).

[44]. E. H. Rattinger, K. Ishida, A. R. Uribe and P. T. Mather. Polymer;54;33503362. (2005).

[45]. S. Turri and M. Levi. Macromolecules 38, 5569-5574.(2005). 
[46]. P. T. Knight, H. Qin and P. T. Mather. Biomacromolecules;9:2458-2467.(2008).

[47]. Liu L, Hu Y, Song L and Nazare S. J Mater Sci; 42:4325-4333. (2007).

[48]. He QL, Song L and Hu Yu. J Mater Sci 44:1308-1316. (2009).

[49]. S. Wu, M. A. Kakimoto and H. Oikawa. Macromolecules;40:5698-5705. (2007).

[50]. M. A. Hoque, S. Shinke, and Y.Kawakami. Macromolecules, 42, 3309-3315. (2009).

[51]. H. Liu and S. Zheng. Macromol Rapid Commun;26:196-200. (2005).

[52]. R. Morent, A. Beaurain P. Dubruel and E. Payen. Prog Org Coat;70:336-341. (2011).

[53]. R. Gu, S. Konar and M. Sain. J Am Oil Chem Soc; 89:2103-2111. (2012).

[54]. S. B. Ghosh, S. B. Ghosh and M. Sain. J Polym Environ;18:437-442. (2010).

[55]. R. A. Vaia and J. F. Maguire. Chem Mater;19:2736-2751. (2007).

[56]. C. Sanchez, B. Julián, P. Belleville and M. Popall. J Mater Chem;15:3559-3592. (2005). 